Pacific Journal of Mathematic 


\title{
ON LOCALLY COMPACT WREATH PRODUCTS
}

\author{
JOHN R. DURBIN
}

\begin{abstract}
The conditions are given under which an algebraic wreath product becomes a topological wreath product with respect to the natural product topology. Various standard topological properties are discussed, and the Haar integral is described. All irreducible representations of the separable locally compact wreath products having a type $I$ group as first factor are determined.
\end{abstract}

1. Introduction. Wreath products have been an important source of examples for (discrete) group theory, as can be seen by their use throughout [7] and [15], for example. Recent work has also shown their usefulness outside group theory ([3], [4], [17]). The possibilities for the theory of arbitrary topological groups seem not to have been openly explored, however. In this paper we begin by recording the condition under which an algebraic wreath product becomes a topological wreath product-with respect to the natural product topology. We next note the conditions under which the product will have various standard topological properties, and we then look at the Haar integral. This brings us to the first problem of substance: to determine the irreducible representations of the locally compact wreath products. This problem has been solved for finite groups through the use of Clifford's work [2] on induced representations (see [9]), and it is natural to make use of induced representations in the more general case as well. The appropriate tool here is Mackey's extension [13] of Clifford's theory, and in the last section we apply this to determine, in particular, all irreducible representations of the separable locally compact wreath products having a type I group as first factor.

2. The groups. If $A$ and $B$ denote groups, then the wreath product of $A$ and $B, A<B$, is constructed as follows. Let $F=A^{B}$ be the direct product of copies of $A$ indexed by the set $B$. Explicitly, $F$ is the set of all functions from $B$ into $A$, made into a group by componentwise multiplication. For $f \in F$ and $b \in B$, define $f^{b} \in F$ by $f^{b}(y)=f\left(y b^{-1}\right)$ for all $y \in B$. Then, for each $b \in B$, the mapping $f \mapsto$ $f^{b}$ is an automorphism of $F$, and the group of all such automorphisms is isomorphic to $B$; the wreath product of $A$ and $B, A<B$, is the extension of $F$ by this group of automorphisms. Each element of $A \prec B$ can be written uniquely as $f b$, with $f \in F$ and $b \in B$, and 
these elements multiply according to the rule $f_{1} b_{1} f_{2} b_{2}=f_{1} f_{2}^{b-1} b_{1} b_{2}$. So $A<B$ is a semi-direct product of $F$ and $B: F \triangleleft A<B, F \cap B=\{e\}$, and $F B=A<B . \quad F$ is called the base group of the wreath product.

The wreath product defined here is sometimes called the standard wreath product, to distinguish it among wreath products in which the second factor is permitted to be an arbitrary permutation group; compare [9]. The standard case appears to bring out the essential ideas, and it avoids the slightly more cumbersome notation of the more general product. (See the remarks following Theorem 2.2, also.)

Now assume $A$ and $B$ to be topological groups; all topological groups will be Hausdorff, and, to avoid degeneracy, we always assume $|A|>1$ and $|B|>1$. Because $A<B$ is a semi-direct product of $F$ and $B$, it is natural to inquire whether $A<B$ becomes a topological group with respect to the product topology on $F \times B$; in other words, whether it is a topological semi-direct product in the usual sense (as in $[6,(6.20)])$. Of course, we must first put a topology on $F=A^{B}$, and for this we take the product topology. It will be convenient to have the following notation: if $S$ is a subset of $A$, and $b \in B$, then $S_{b}$ will denote $\{f \in F: f(b) \in S$ and $f(x)=e$ for $x \neq b\}$.

Theorem 2.1. If $A$ and $B$ are topological groups, then $A<B$ is a topological group with respect to the product topologies on $F=A^{B}$ and on $F \times B$ iff $B$ is discrete.

Proof. For $A<B$ to be a topological group, it is necessary and sufficient that the map $(f, b) \mapsto f^{b}$ of $F \times B$ into $F$ be continuous. For each $x \in B$, let $\varphi_{x}: F \times B \rightarrow A_{x}$ be defined by $\varphi_{x}(f, b)=f^{b}(x)$. Then $A \prec B$ will be a topological group iff each $\varphi_{x}$ is continuous, and thus iff

$$
\varphi_{x}^{-1}\left(U_{x}\right)=\bigcup_{b \in B}\left[\left(U_{x b^{-1}} \times A^{B \backslash\left\{x b^{-1\}}\right.}\right) \times\{b\}\right]
$$

is open for each open set $U$ of $A$ and each $x \in B$. This is certainly the case if $B$ is discrete. In proving the converse it suffices to show that $B$ must have a finite open subset, for $B$ is Hausdorff and homogeneous. Suppose, then, that each $\varphi_{x}$ is continuous, so that in particular $\varphi_{e}$ is continuous. Choose a nonempty proper open subset $U$ of $A$, and choose $f b \in \varphi_{e}^{-1}\left(U_{e}\right)$. Then there are open subsets $V_{1}$ in $F$ and $V_{2}$ in $B$ such that $f b \in V_{1} \times V_{2} \subseteq \varphi_{e}^{-1}\left(U_{e}\right)$. Furthermore, it can be assumed that there are elements $b_{1}, \cdots, b_{k}$ in $B$ and open sets $W_{1}, \cdots, W_{k}$ in $A$ such that $f\left(b_{j}\right) \in W_{j}$ for $1 \leqq j \leqq k$ and $V_{1}=W_{1} \times \cdots \times W_{k} \times$ $A^{\left.B \backslash \backslash b_{1}, \cdots, b_{k}\right\}}$. But then for each $b^{\prime} \in V_{2}$ we must have $V_{1} \times\left\{b^{\prime}\right\} \subseteq V_{1} \times V_{2} \subseteq$ $\varphi_{e}^{-1}\left(U_{e}\right)$, and thus $V_{1} \leqq U_{b^{\prime}-1} \times A^{B \backslash\left\langle b^{\prime}-1\right\}}$. But the latter is impossible 
for some $b^{\prime}$ if $V_{2}$ is infinite, as can be seen by choosing $b^{\prime} \notin\left\{b_{1}^{-1}, \ldots\right.$, $\left.b_{k}^{-1}\right\}$. Thus the open set $V_{2}$ of $B$ must be finite. Finally, we note that $F \times B$ is Hausdorff since it is a product of Hausdorff spaces.

By the wreath product of topological groups we shall always mean the product referred to in Theorem 2.1. The next theorem follows readily from standard facts about product spaces and the topological properties involved.

Theorem 2.2. Let $A$ and $B$ denote topological groups. (a) $A<B$ is compact iff $A$ is compact and $B$ is finite. (b) $A$ < $B$ is locally compact iff either $A$ is locally compact and $B$ is finite, or $A$ is compact and $B$ is arbitrary (but discrete). (c) $A<B$ is separable (has a countable open base) iff $A$ is separable and $B$ is countable.

In (discrete) group theory it is often useful to consider, in place of $A<B$, the restricted (standard) wreath product. This is constructed by using $A^{(B)}=\left\{f: f \in A^{B}\right.$ and $f(x) \neq e$ for at most finitely many $x \in B\}$, rather than $A^{B}$, for the base group. This restricted wreath product is a subgroup of $A<B$, and is, of course, a topological group with the relative topology. However, when one is interested only in locally compact groups, as we are in studying representations, such a restricted group yields nothing new, for it will be locally compact only if $B$ is finite, in which case it is the same as $A<B$.

Philip Hall was the first to show the usefulness of wreath products for the theory of infinite discrete groups, making use of the "generalized" wreath product, $W r\left\{H_{\lambda}: \lambda \in \Lambda\right\}$, constructed with arbitrary ordered set $\Lambda$ and arbitrary transitive permutation groups $H_{\lambda}$; see [5] as well as references to Hall's paper in [15]. It follows readily from the law of segmentation [5, p. 172] for general wreath products, together with Theorem 2.1, that such a wreath product will become a topological group in our context only if $\Lambda$ has a least element $\mu$ and all $H_{2}$ for $\lambda \neq \mu$ are discrete. With $A=H_{n}$ and $B=W r\left\{H_{\lambda}\right.$ : $\lambda \neq \mu\}$, the group will have the form $A<B$, and thus will, in fact, be in the class we are considering.

Any extension of a discrete group $A$ by a discrete group $B$ can be embedded in the discrete wreath product $A<B$. Lakshmi [10] considered the corresponding statement for topological groups, obtaining an analogous theorem with suitable conditions on the groups, and with the compact-open topology, rather than the product topology, on the base group of the wreath product. His theorem implies that if $A$ is locally compact and $B$ is discrete, then any topological extension of $A$ by $B$ can be embedded in (our) $A<B$.

Finally, we point out that an example of Mackey's [13, pp. 309- 
310], showing that a semi-direct product need not have trivial "Mackey obstruction", is a variation on a wreath product.

3. The Haar integral. We now describe how the Haar integral for locally compact $A$ < $B$ can be given explicitly in terms of the Haar integrals for its component subgroups. We make use of the known characterizations of the Haar integral for arbitrary direct and semidirect products.

First consider $F=A^{B}$, the base group. Because of Theorem 2.2(b), there are two cases to consider: (1) A locally compact and $B$ finite, and (2) $A$ compact and $B$ arbitrary (discrete). In case (1), Fubini's Theorem and induction yield that the right Haar measure on $F$ is that corresponding to the product of $|B|$ copies of the right Haar measure on $A$ :

$$
\int g(f) d f=\int \cdots \iint g\left(a_{1}, a_{2}, \cdots, a_{n}\right) d a_{1} d a_{2} \cdots d a_{n},
$$

where $d a_{1}, \cdots, d a_{n}$ correspond one-to-one with the elements of $B$, the order being irrelevant, and where each $d a_{j}$ designates Haar measure for the corresponding copy of $A$ in the base group [6, (13.16) and (15.29)]. Case (2) is similar, although in general involving nets and thus slightly more complicated; the details follow from [6, (13.8) and (15.17)(j)].

With $F$ taken care of, we are ready to look at $A<B$ considered as a semidirect product of $F$ and $B$. It is easy to show [6, (15.29)] that the right Haar integral for a semi-direct product simply corresponds to the right Haar measures for the two component groups. We formalize this for $A<B$ as follows.

THEOREM 3.1. Let $d w, d f, d b$ designate Haar measure on $W$ $A<B, F=A^{B}$, and $B$, respectively. Then

$$
\int g(w) d w=\iint g(f b) d f d b
$$

for each function $g$ with compact support on $W$, with $d f$ as described accompanying (3.1).

There remains the question of the modular function. For $\mu$ and $\nu$ left and right invariant Haar measures on $W=A<B$, respectively, we take $\Delta_{W}$ to be the function given by

$$
\int g(w) d \nu(w)=\int \Delta_{w}(w) g(w) d \mu(w)
$$

(for appropriately chosen factors of proportionality for $\mu$ and $\nu$ ). In 
the same way, we get the modular functions $\Delta_{A}, \Delta_{F}$, and $\Delta_{B}$ for $A$, $F$, and $B$.

THEOREM 3.2. If $B$ is infinite, then $A<B$ is unimodular. If $B$ is finite, then $A<B$ is unimodular iff $A$ is unimodular. In any case, for $B$ finite, the modular function $\Delta_{W}$ for $W=A<B$ is given by

$$
\Delta_{W}(f b)=\Delta_{F}(f)=\prod_{x \in B} \Delta_{A} f(x) .
$$

Proof. We require, tentatively, the function $\delta$ determined as follows.

Fact 3.1 [6, (15.29)]. If $\alpha$ is an automorphism of a locally compact group $H$, and $\nu$ is a right invariant Haar measure on $H$, then there exists a unique real number $\delta(\alpha)>0$ such that

$$
\int g\left(\alpha^{-1}(x)\right) d \nu(x)=\delta(\alpha) \int g(x) d \nu(x)
$$

for each function $g$ with compact support on $H$.

We now recall that each $b \in B$ determines an automorphism $\bar{b}: f \mapsto$ $f^{b}$ of $F$. Applying [6, (15.29)], we find that

$$
\Delta_{W}(f b)=\delta(\bar{b}) \Delta_{F}(f) \Delta_{B}(b)
$$

for all $f b \in A<B(f \in F, b \in B)$. But $\bar{b}$ merely permutes the components of each $f \in F$, and thus, taking into account the irrelevance of the order of the $d a_{j}$ in (3.1) (and the corresponding fact for $B$ infinite), we see that in our case (3.2) takes the form

$$
\int g(f) d f=\int g\left(\bar{b}^{-1}(f)\right) d f=\delta(\bar{b}) \int g(f) d f .
$$

That is, $\delta(\bar{b}) \equiv 1$. Furthermore, $B$ is discrete and therefore unimodular, so that $\Delta_{B}(b) \equiv 1$ as well. Thus (3.3) reduces to $\Delta_{W}(f b)=\Delta_{F}(f)$. In case $B$ is infinite, then $A$ must be compact (Theorem 2.2(b)), whence $F$ is compact and therefore unimodular, and so $\Delta_{W}(f b)=\Delta_{F}(f) \equiv 1$. So the question of nontrivial unimodular function arises only when $B$ is finite, and then [6, (15.29)(a)], induction, and the special nature of direct products as semi-direct products yield

$$
\Delta_{W}(f b)=\Delta_{F}(f)=\prod_{x \in B} \Delta_{A} f(x) .
$$

Noting that if $B$ is infinite then $A$ must be compact and hence unimodular, we have the following corollary.

Corollary. $A<B$ is unimodular iff $A$ is unimodular. 
4. Representations. The appropriate tool for use in determining the irreducible representations of the groups $A<B$ is, as noted in the Introduction, the theory of induced representations. The irreducible representations of the base group can be described in terms of the irreducible representations of $A$. These irreducible representations of the base group can then be used to generate representations of $A$ < $B$ by induction from an intermediate subgroup. There are two questions: (1) Is every representation of $A<B$ obtained in this way irreducible? (2) Does every irreducible representation of $A$ < $B$ appear as such an induced representation? In the case of finite groups this program is completely successful: the answer to both questions is Yes. We shall see in this section that the program can be shown to be successful for a much larger class of separable locally compact $A<B$. For the finite case see [16], [1], [7, pp. 583-585], [8], [9].

We consider groups that are separable and locally compact; the wreath products in this class are specified by Theorem 2.2. We also assume $A$ to be of type I: this includes the possibilities that $A$ is compact, or Abelian, or a semi-simple Lie group, or discrete and Abelian-by-finite. We assume acquaintance with Mackey's papers on induced representations, especially [11]. By a representation of a group $N$ we mean a continuous homomorphism $x \mapsto L_{x}$ of $N$ into the group of all unitary transformations of a separable Hilbert space $H(L)$ onto itself, where continuity means that for each $\psi \in H(L)$ the function $x \mapsto L_{x}(\varphi)$ is continuous from $N$ into $H(L)$. If $N$ is a closed subgroup of a group $G$, then $L \uparrow G$ will denote the representation of $G$ induced by the representation $L$ of $N$. If $G$ is a group, then $\widehat{G}$ will denote a set of irreducible representations of $G$, one from each equivalence class.

We begin by considering the base group, $F=A^{B}$. Its irreducible representations are the tensor products of those of its component subgroups $A_{b}$; let us make this explicit.

LEMma 4.1. There is a one correspondence between the set $\hat{F}$ and the set of all maps $\mu: B \rightarrow \hat{A}$ having the property that $\mu(b)$ is the trivial representation almost always. Given $\mu$, the corresponding representation $L$ of $F$ is defined by

$$
L_{f}=\mu\left(b_{1}\right)_{f\left(b_{1}\right)} \otimes \cdots \otimes \mu\left(b_{n}\right)_{f\left(b_{n}\right)},
$$

where $\mu(b)$ is trivial for $b \neq b_{1}, \cdots, b_{n}$.

Proof. If $B$ is infinite, then $A$ must be compact, and this is [6, II, (27.43)]. If $B$ is finite, then $A$ is (still) of type I, and the result follows from [11, pp. 199-200]. 
Consider the action of $B$ on $\hat{F}$ defined as follows: for $L \in \hat{F}$ and $b \in B, L \mapsto L^{b}$, where $L_{f}^{b}=L_{b f^{b}-1}=L_{f^{b-1}}$. Then, for each $L \in \hat{F}$, the stability factor of $L$ in $B$ is defined to be

$$
H_{L}=\left\{b \in B: L^{b} \sim L\right\},
$$

where $\sim$ denotes equivalence. The stability group of $L$ is $F H_{L}$. We can characterize the stability factors of elements of $\hat{F}$ by using the following notion.

Definition. Let $H$ denote a subgroup of $B$. A function $\mu$ on $B$ is barely constant on the (left) cosets of $H$ if

$$
\mu\left(b_{i}\right)=\mu\left(b_{j}\right) \quad \text { whenever } \quad b_{j} b_{i}^{-1} \in H
$$

but if (4.1) does not hold with $H$ replaced by any subgroup of $B$ strictly containing $H$.

Lemma 4.2. Assume $\mu: B \rightarrow \hat{A}$, trivial almost always. Then $\mu$ is barely constant on the cosets of $H$ iff $H$ is the stability factor for the representation $L$ (of $F$ ) corresponding to $\mu$.

Proof. Let $L$ denote the representation corresponding to $\mu$ as in Lemma 4.1. Typical components of $L_{f}$ and $L_{f}^{b}$ will be $\mu\left(b_{i}\right)_{f\left(b_{i}\right)}$ and $\mu\left(b_{j}\right)_{f\left(b_{j} b\right)}$, respectively. For $B$ infinite, we can argue as in [8] (or [7, pp. 583-585]) merely extending from finite to arbitrary compact groups. We see that if $\mu$ is barely constant on the cosets of $H$, and if $b \in H$, then $\mu\left(b_{i}\right)=\mu\left(b_{j}\right)$ when $b_{i}=b_{j} b$; thus $L$ and $L^{b}$ have equal characters and are, therefore, equivalent [6, II, (27.32)]. Assume, conversely, that $b$ is in the stability factor for $L$, and choose $f \in F$ with $f(x)=e$ for $x \neq b_{k}$ (any fixed $k$ ). Take characters, and deduce that

$$
\operatorname{tr} \mu\left(b_{k}\right)_{f\left(b_{k}\right)} \cdot \operatorname{deg} \mu\left(b_{k} b^{-1}\right)=\operatorname{tr} \mu\left(b_{k} b^{-1}\right)_{f\left(b_{k}\right)} \cdot \operatorname{deg} \mu\left(b_{k}\right)
$$

for all $f\left(b_{k}\right) \in A$. Using [6, II, (27.30)], this implies that $\mu\left(b_{k}\right)$ and $\mu\left(b_{k} b^{-1}\right)$ must be equivalent. Thus $\mu$ must be constant on the cosets of $\langle b\rangle$, and the lemma is established for infinite $B$. If $B$ is finite, the lemma is a consequence of [11, pp. 199-200].

We must next extend representations of $F$ to representations of their stability groups. This can always be done [13], but it might necessitate the introduction of projective representations corresponding to nontrivial multipliers, even in the case of semi-direct products; in fact, as pointed out at the end of $\S 3$, Mackey has used a variation on a wreath product to illustrate this point. In our case, however, 
projective representations are not required, as the next result shows.

LEMmA 4.3. (Specht). Each $L \in \hat{F}$ can be extended to $L^{\prime} \in \widehat{F H}$. (The Mackey obstruction is trivial.)

Proof. With $L$ as in Lemma 4.1 , each $b_{k}$-component can be represented in matrix form with entries $\mu\left(b_{k}\right)_{f\left(b_{k}\right)}\left(\alpha_{k}, \beta_{k}\right)[14$, pp. 112114]. Then $L_{f}$ will be represented by the matrix with $\alpha_{1} \beta_{1} \ldots \alpha_{n} \beta_{n}$ entry as indicated:

$$
\left(u\left(b_{1}\right)_{f\left(b_{1}\right)}\left(\alpha_{1}, \beta_{1}\right) \cdots \mu\left(b_{n}\right)_{f\left(b_{n}\right)}\left(\alpha_{n}, \beta_{n}\right) .\right.
$$

Following Specht $\left[16\right.$, p. 330], the desired representation $L^{\prime}$ will have matrix form with the $\alpha_{1} \beta_{1} \ldots \alpha_{n} \beta_{n}$ entry of $L_{f b}^{\prime}$ as indicated:

$$
\left(\mu\left(b_{1}\right)_{f\left(b_{1}\right)}\left(\alpha_{1}, \beta_{1}^{\prime}\right) \cdots \mu\left(b_{n}\right)_{f\left(b_{n}\right)}\left(\alpha_{n}, \beta_{n}^{\prime}\right)\right),
$$

for $\beta_{i}^{\prime}=\beta_{j}$ when $b_{j}=b_{i} b^{-1}$. Then $L^{\prime} \downarrow F=L$, so that $L^{\prime}$ is irreducible because $L$ is irreducible.

If $M$ is a representation of $H_{L}$, and $\pi_{L}: F H_{L} \rightarrow F H_{L} / F \cong H_{L}$ is the natural homomorphism, then we can form the representation $L^{\prime} \otimes$ $M \pi_{L}$ of $F H_{L}$. We can now state our main theorem on representations. The terminology is that of [13].

Theorem 4.1. Assume $A$ of type $I$, and $A>B$ separable and locally compact. Then the correspondence $M \leftrightarrow\left(L^{\prime} \otimes M \pi_{L}\right) \uparrow(A<B)$ is one-one between the of set primary representations of $H_{L}$ and the set of primary representations of $A<B$ which yield a multiple of $L$ on restriction to $F$. The correspondence preserves irreducibility and type.

Proof. The theorem is a consequence of the preceding lemmas and Theorem 8.4 of [13], if only we verify that $F$ is regularly embedded in $A<B$. However, $F$ is of type $I$ and thus $\hat{F}$ is standard, and so Theorem 9.2 of [13] applies; that there is a Borel set meeting each orbit exactly once can be seen from [12, p. 64] and [13, p. 294].

CoROLlaRY 4.1. The equivalence classes of irreducible representations of $A<B$ are in one-one correspondence with the set of triples $(H, \varphi, M)$, where $H$ denotes a finite subgroup of $B, \varphi \rightarrow \hat{A}$ is trivial almost always and barely constant on the cosets of $H$, and $M \in \hat{H}$, provided functions $\varphi, \psi: B \rightarrow \hat{A}$ are identified whenever the corresponding representations of $F$ are conjugate (that is, whenever $\varphi \leftrightarrow K$ and $\psi \leftrightarrow L$ and $K^{b}=L$ for some $b \in B$ ). 
COROLLARY 4.2. If $B$ is torsion-free then all irreducible representations of $A<B$ are induced from representions of the base group.

Corollary 4.3. If $A$ is of type $I$, then $A \backslash B$ is of type I.

Proof. This is a consequence of Theorem 9.3 of [13], if we note that all stability factors $H_{L}$ are finite and thus of type $I$.

Corollary 4.4. All irreducible representations of $A \backslash B$ are finite dimensional iff $B$ is finite and all irreducible representations of $A$ are finite dimensional.

\section{REFERENCES}

1. N. N. Aizenberg, On the representations of the wreath products of finite groups, (Russian, English summary.), Ukrain. Mat. Z. no. 4, 13 (1961), 5-12.

2. A. H. Clifford, Representations induced in an invariant subgroup, Ann. of Math., 38 (1937), 533-550.

3. C. F. Dunkl and D. E. Ramirez, Krawtchouk polynomials and the symmetrization of hypergroups, SIAM J. Math. Anal., 5 (1974), 351-366.

4. G. Eagleson, A characterization theorem for positive definite sequences on the Krawtchouk polynomials, Austral. J. Statist., 11 (1969), 29-38.

5. P. Hall, Wreath powers and characteristically simple groups, Proc. Cambridge Philos. Soc., 58 (1962), 170-184.

6. E. Hewitt and K. A. Ross, Abstract Harmonic Analysis, Springer-Verlag, BerlinNew York, Vol. I, 1963; Vol. II, 1970.

7. B. Huppert, Endliche Gruppen I, Springer-Verlag, Berlin-New York, 1967.

8. A. Kerber, Zur Darstellungstheorie von Kranzprodukten, Canad. J. Math., 20 (1968), 665-672.

9. — Representations of Permutation Groups, Lecture Notes in Mathematics, Vol. 240, Springer-Verlag, Berlin-New York, 1971.

10. A. Lakshmi, Topological wreath products, J. Austral. Math. Soc., 5 (1965), 396-400.

11. G. W. Mackey, Induced representations of locally compact groups. II, Ann. of Math., (2) 58 (1953), 193-221.

12. - The Theory of Group Representations, University of Chicago, 1955.

13. - Unitary representations of group extensions. I, Acta Math. 99 (1958), 265-311.

14. M. A. Naimark, Normed Rings, trans. by Leo F. Boron, Wolters-Noordhoff Publishing, Groningen, The Netherlands, 1970.

15. D. J. S. Robinson, Finiteness Conditions and Generalized Soluble Groups, SpringerVerlag, Berlin-New York, Part 1, 1972; Part 2, 1972.

16. W. Specht, Eine Verallgemeinerung der Permutationsgruppen, Math. Zeit., 37 (1933), 321-341.

17. D. Vere-Jones, Finite bivariate distributions and semigroups of nonnegative matrices, Quart. J. Math., Oxford (2), 22 (1971), 247-270.

Received November 1, 1974.

The University of TeXas-Austin 



\title{
PACIFIC JOURNAL OF MATHEMATICS
}

\author{
EDITORS
}

RICHARD ARENS (Managing Editor)

University of California

Los Angeles, California 90024

R. A. Beaumont

University of Washington

Seattle, Washington 98105
J. DugundJI

Department of Mathematics University of Southern California Los Angeles, California 90007

D. Gilbarg and J. Milgram

Stanford University

Stanford, California 94305

\section{ASSOCIATE EDITORS}

E. F. BECKENBACH

B. H. NeUManN

F. WOLF

K. YosHIDA

\section{SUPPORTING INSTITUTIONS}

UNIVERSITY OF BRITISH COLUMBIA

GALIFORNIA INSTITUTE OF TECHNOLOGY

UNIVERSITY OF CALIFORNIA

MONTANA STATE UNIVERSITY

UNIVERSITY OF NEVADA

NEW MEXICO STATE UNIVERSITY

OREGON STATE UNIVERSITY

UNIVERSITY OF OREGON

OSAKA UNIVERSITY
UNIVERSITY OF SOUTHERN CALIFORNIA

STANFORD UNIVERSITY

UNIVERSITY OF TOKYO

UNIVERSITY OF UTAH

WASHINGTON STATE UNIVERSITY

UNIVERSITY OF WASHINGTON

AMERICAN MATHEMATICAL SOCIETY NAVAL WEAPONS CENTER 


\section{Pacific Journal of Mathematics}

\section{Vol. 57, No. $1 \quad$ January, 1975}

Keith Roy Allen, Dendritic compactification ......................... 1

Daniel D. Anderson, The Krull intersection theorem ..................... 11

George Phillip Barker and David Hilding Carlson, Cones of diagonally dominant

matrices .............................................

David Wilmot Barnette, Generalized combinatorial cells and facet splitting ........

Stefan Bergman, Bounds for distortion in pseudoconformal mappings ............

Nguyên Phuong Các, On bounded solutions of a strongly nonlinear elliptic

equation ...............................................

Philip Throop Church and James Timourian, Maps with 0-dimensional critical

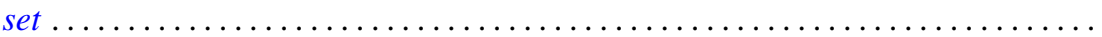

G. Coquet and J. C. Dupin, Sur les convexes ubiquitaires ................. 67

Kandiah Dayanithy, On perturbation of differential operators............... 85

Thomas P. Dence, A Lebesgue decomposition for vector valued additive set

functions .............................................. 91

John Riley Durbin, On locally compact wreath products.................. 99

Allan L. Edelson, The converse to a theorem of Conner and Floyd ............. 109

William Alan Feldman and James Franklin Porter, Compact convergence and the

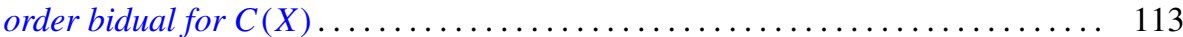

Ralph S. Freese, Ideal lattices of lattices......................... 125

R. Gow, Groups whose irreducible character degrees are ordered by divisibility ... 135

David G. Green, The lattice of congruences on an inverse semigroup ............ 141

John William Green, Completion and semicompletion of Moore spaces .......... 153

David James Hallenbeck, Convex hulls and extreme points of families of starlike and close-to-convex mappings .................................. 167

Israel (Yitzchak) Nathan Herstein, On a theorem of Brauer-Cartan-Hua type ...... 177

Virgil Dwight House, Jr., Countable products of generalized countably compact spaces ............................................ 183

John Sollion Hsia, Spinor norms of local integral rotations. I . . . . . . ......... 199

Hugo Junghenn, Almost periodic compactifications of transformation

semigroups....................................

Shin'ichi Kinoshita, On elementary ideals of projective planes in the 4-sphere and oriented $\Theta$-curves in the 3 -sphere ............................ 217

Ronald Fred Levy, Showering spaces............................ 223

Geoffrey Mason, Two theorems on groups of characteristic 2-type............. 233

Cyril Nasim, An inversion formula for Hankel transform ................... 255

W. P. Novinger, Real parts of uniform algebras on the circle ................ 259

T. Parthasarathy and T. E. S. Raghavan, Equilibria of continuous two-person

games............................................... 265

John Pfaltzgraff and Ted Joe Suffridge, Close-to-starlike holomorphic functions of several variables ....................................... 271

Esther Portnoy, Developable surfaces in hyperbolic space .................. 281

Maxwell Alexander Rosenlicht, Differential extension fields of exponential type . . . 289

Keith William Schrader and James Lewis Thornburg, Sufficient conditions for the

existence of convergent subsequences ........................... 301

Joseph M. Weinstein, Reconstructing colored graphs .................. 307 